\title{
Effects of Preoperative Anxiety on Postoperative Delirium in Elderly Patients Undergoing Elective Orthopedic Surgery: A Prospective Observational Cohort Study
}

This article was published in the following Dove Press journal:

Clinical Interventions in Aging

\section{Aolin Ren ${ }^{1, *}$ \\ Na Zhang ${ }^{1, *}$ \\ He Zhu (D) ${ }^{\prime}$ \\ Kang Zhou' \\ Yuan Cao' \\ Jindong Liu ${ }^{1,2}$}

'Department of Anesthesiology, Affiliated Hospital of Xuzhou Medical University, Xuzhou, Jiangsu, People's Republic of China; ${ }^{2}$ Jiangsu Province Key Laboratory of Anesthesiology, Xuzhou Medical University, Xuzhou, Jiangsu, People's Republic of China

*These authors contributed equally to this work
Correspondence: Jindong Liu Department of Anesthesiology, Affiliated Hospital of Xuzhou Medical University,

Xuzhou Medical University, Huaihai West Road \#99, Xuzhou 221000, People's

Republic of China

Tel +86 $108580-2333$

Email liujindong1818@I63.com
Background: Postoperative delirium (POD) is common and has negative effects on elderly patients. There is a critical need to identify patients at high risk of POD so that providers can better offer targeted interventions in the preoperative and intraoperative periods. We aimed to characterize the prevalence of preoperative anxiety and investigate whether preoperative anxiety predicted the onset of POD in elderly patients undergoing elective orthopedic surgery. Methods: We conducted a prospective observational cohort study of elderly patients (aged 65 years or older) undergoing elective orthopedic surgery. Preoperative anxiety was assessed using the Hospital Anxiety and Depression Scale-Anxiety (HADS-A), with clinically significant values defined as HADS-A $>7$. POD was diagnosed according to the Confusion Assessment Method (CAM) criteria on the 5 postoperative days. To determine the independent risk factors for POD, multivariable logistic regression was conducted, including those variables with a $\mathrm{p}$-value $<0.05$ at univariate analysis.

Results: There were 263 patients included in the study. Seventy-three (27.8\%) patients were diagnosed to be POD. Median duration of delirium was 2 days. Forty (15.2\%) patients were assessed to experience preoperative anxiety. The occurrence of preoperative anxiety in total hip arthroplasty (THA), proximal femoral nail antirotation (PFNA), and total knee arthroplasty (TKA) was $12.5 \%, 16.1 \%$, and $19.5 \%$, respectively. Based on multivariable analysis, only age (odds ratio $[\mathrm{OR}]=1.099,95 \%$ Confidence Interval $[\mathrm{CI}]: 1.013-1.192 ; P=0.023$ ), Mini Mental State Examination (MMSE) (OR=0.603, 95\% CI: 0.432-0.842; $P=0.003$ ) and preoperative anxiety $(\mathrm{OR}=3.119,95 \% \mathrm{CI}: 1.144-8.500 ; P=0.026)$ were independently associated with POD.

Conclusion: In conclusion, the current study reveals that preoperative anxiety helps to predict the risk of POD in elderly patients undergoing elective orthopedic surgery. Relieving preoperative anxiety could be a new target for preventive interventions to reduce POD.

Keywords: delirium, anxiety, orthopedic, risk factors

\section{Introduction}

POD, characterized by acute disturbance of consciousness, attention, cognition, and perception, ${ }^{1}$ is a common postoperative complication in elderly patients undergoing orthopedic surgery. The incidence of POD was found to be $3.6-28.3 \%$ in elective orthopedic surgery and up to $53.3 \%$ in hip fracture samples. ${ }^{2}$ Delirium is associated with postoperative neurocognitive disorder impairment, prolonged hospital stays, 
increased health care costs, and elevated mortality rate. ${ }^{3-6}$ It is essential to control and/or eliminate risk factors for the prevention of delirium with the aging of the population.

The cause of delirium is thought to be multifactorial. There is a consensus that several unmodifiable risk factors are significantly associated with POD including cognitive impairment, comorbidity, sensorial deficits, malnutrition, impaired functional status, and frailty. ${ }^{7,8}$ It is estimated that $30 \%-40 \%$ of cases of delirium are preventable. ${ }^{9}$ Multi-component interventions targeted at modifiable risk factors have been shown to reduce delirium in hospitalized patients. ${ }^{10,11}$ One such risk factor may be preoperative psychiatric symptoms ${ }^{12-18}$ and the evaluation of psychological status has often been overshadowed by the focus on multiple comorbidities in the elderly. ${ }^{12}$ Anxiety disorders, one of the outstanding psychiatric symptoms, are highly prevalent among the aged. ${ }^{19}$ Preoperative anxiety is defined as an unpleasant state of uneasiness or tension that is secondary to a patient being concerned about a disease, hospitalization, anesthesia, and surgery, or the unknown. ${ }^{20}$ Studies exploring the association between preoperative anxiety and POD are few and agreement differs among different populations. ${ }^{13,15,16,18}$ Therefore, it deserves further investigation.

In the present study, we investigated whether preoperative anxiety would predict the onset of POD in elderly patients undergoing elective orthopedic surgery. Besides, the prevalence of preoperative anxiety in specific orthopedic procedures is described.

\section{Methods}

\section{Study Design}

This study was approved by the Ethics Committee of the Affiliated Hospital of Xuzhou Medical University (Certification No. XYFY2020-KL114-01) and registered at https://www.clinicaltrials.gov (clinical trial registration number: NCT04216615). The clinical trial was conducted in accordance with the Declaration of Helsinki. All participants provided informed written consent. The study is reported according to the criteria set out in the Strengthening the Reporting of Observational Studies in Epidemiology (STROBE) checklist. ${ }^{21}$

\section{Study Population}

We performed a single-center prospective, observational study on elderly patients (aged 65 years or older) with American Society of Anesthesiologists (ASA) I-III undergoing elective orthopedic surgery. Patients were excluded if they met any of the following criteria: a history of craniocerebral trauma surgery or severe central nervous system disease; cannot complete neuropsychological testing; a history of neurological disease (eg, Alzheimer's disease, Parkinson's syndrome); delirium occurred during preoperative assessment; inability to read or understand the informed consent documents.

\section{Measures}

\section{Demographic and Clinical Characteristics}

We used standardized and structured questionnaires to collect demographic data, including age, sex, and socioeconomic variables. Clinical characteristics (laboratory tests) were obtained from patients' medical records.

\section{Preoperative Anxiety}

Preoperative anxiety was measured using the HADS-A, a standardized, self-report instrument consisting of 7 items. The Hospital Anxiety and Depression Scale (HADS) is a frequently used self-rating scale developed to assess psychological distress in non-psychiatric patients. $^{22}$ HADS has good reliability and validity, which can be used to evaluate anxiety and depression in general hospitals. $^{23}$ The presence of preoperative anxiety was defined as a HADS-A score of 8 or more.

\section{Postoperative Delirium}

Delirium was diagnosed according to the CAM criteria, which defines delirium present if a patient exhibits an acute change or fluctuation in mental status, inattention, and either disorganized thinking or an altered level of consciousness. $^{24}$ For patients admitted to ICU after surgery, we identified delirium using the validated Richmond Agitation Sedation Scale (RASS) and Confusion Assessment Method for the ICU (CAM-ICU). ${ }^{25}$

\section{Other Risk Factors of POD}

Based on current state-of-the-art evidence and guidelines, ${ }^{7}$ the following data were collected: body mass index (BMI), history of smoking, alcohol use, ASA classification, CCI (Charlson Comorbidity Index), VAS (visual analogue scale) pain score, cognitive function assessed using the MMSE. Eleven intraoperative and postoperative variables including type of surgery, duration of anesthesia, duration of surgery, blood transfusion, total fluid administration, nerve block, corticosteroid, dexmedetomidine, benzodiazepines, patient-controlled intravenous analgesia (PCIA), and ICU admission were also recorded. 


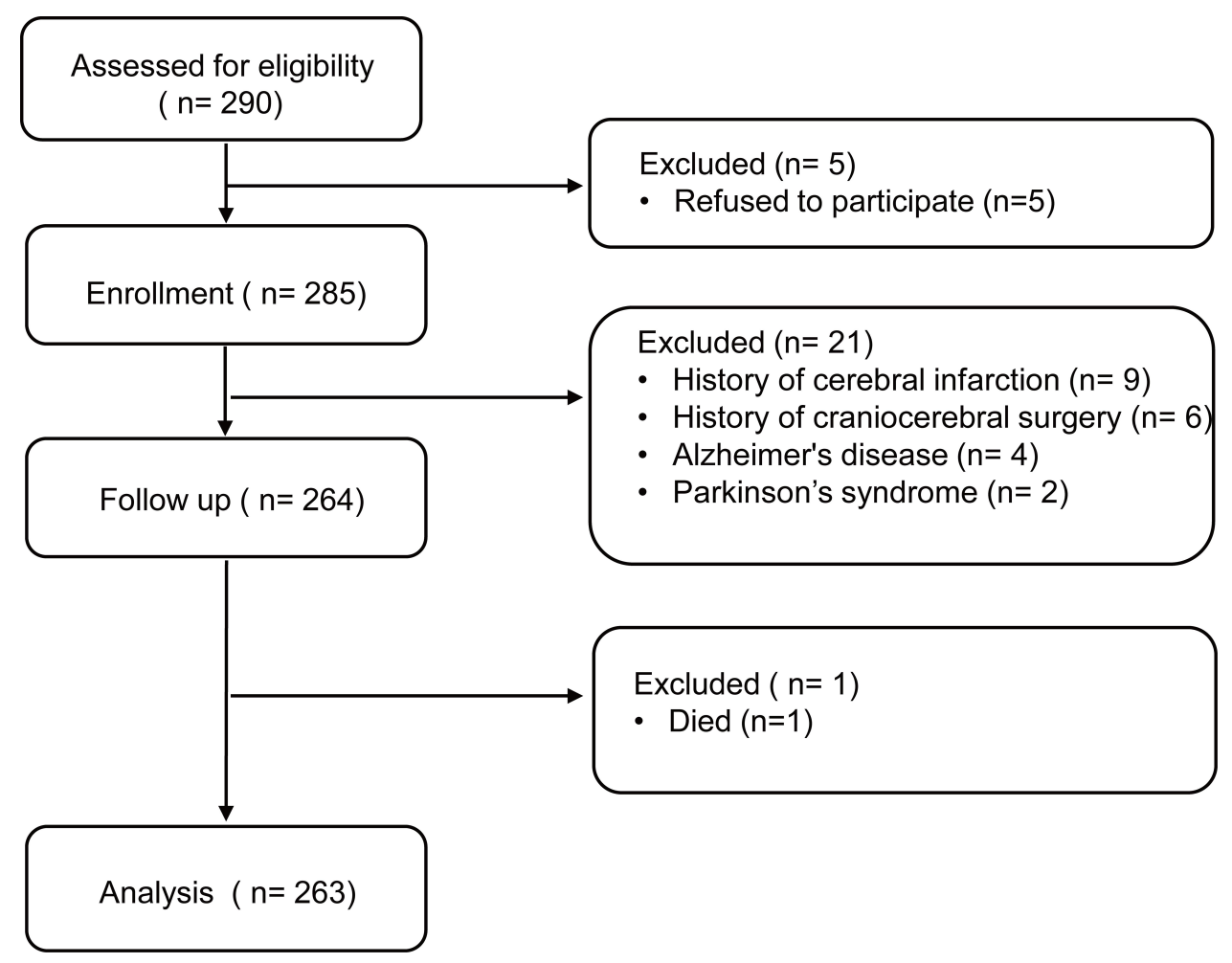

Figure I Flowchart for participant enrollment.

\section{Anesthetic Procedure and Course of the Study}

All patients received standard monitoring, which included electrocardiography, invasive blood pressure assessment, and pulse oximetry after entering the operating room. After general anesthesia induction and tracheal intubation, lungs were ventilated mechanically to maintain an endtidal carbon dioxide of 35-45 $\mathrm{mmHg}$. Anesthesia was maintained via inhaled sevoflurane, propofol, intravenous remifentanil to reach a Bispectral Index (BIS) value of 40 to 60 throughout the procedure. Anesthesiologists strived to maintain hemodynamic stability with the administration of vasopressors and/or inotropics, fluid therapy, and red blood cell (RBC) transfusion.

\section{Statistical Analyses}

Statistical analyses were performed using IBM SPSS Statistics 25. Descriptive statistics are presented as absolute frequency (number) and relative frequency (percentage) for categorical variables, and as mean and standard deviation (SD) for continuous variables. The distribution of continuous variables was checked using the Kolmogorov-Smirnov test of normality. Differences between groups were examined using Student's $t$ test, Mann-Whitney $U$-test, or Chi-square test, according to the type of variables and their distribution. The influence of different variables on outcome variables was assessed in logistic regression models. Variables with a $P<0.05$ in univariable analyses were included in the multivariable logistic regression model to control confounding factors. We used variance inflation factors (VIFs) to examine possible collinearity among covariates. Preoperative anxiety was added to the multivariate model as a variable of interest. We checked the model fitness for the logistic regression using Hosmer and Lemeshow goodness-of-fit test statistics. A p-value less than 0.05 was considered statistically significant.

\section{Results}

\section{Sample}

During the study period, 290 patients were assessed for eligibility, of whom 285 (98.2\%) agreed to participate (Figure 1). Among those screened, 21 were excluded from the study for various reasons, including 9 had a history of cerebral infarction, 6 had a history of craniocerebral surgery, 4 of Alzheimer's disease, and 2 of Parkinson's syndrome. One participant was lost to followup as the patient died after the operation due to fatal 
pulmonary embolism. Finally, a total of 263 patients were included in the study. The mean age of patients was 74.2 years, $74(28.1 \%)$ were male (Table 1). Our study included three types of surgery: THA, PFNA $(45.6 \%$ and $21.3 \%$, respectively), and TKA (33.1\%) (Table 2).

\section{Incidence and Duration of Postoperative}

\section{Delirium}

In the whole sample, $73(27.8 \%)$ patients were diagnosed to be delirium in the 1 to 5 days after the operation. On postoperative day 1,3 , and 5, the incidence of POD was $10.6 \%, 14.8 \%$, and $6.5 \%$, respectively. The median duration of delirium was 2 days. The incidence of POD in THA, PFNA, and TKA was $31.7 \%, 30.4 \%$, and $20.7 \%$, respectively (Figure 2). Delirious patients had longer postoperative length of hospital stays than non-delirious patients (Table 2).

\section{The Occurrence of Preoperative Anxiety}

Forty $(15.2 \%)$ patients were assessed to experience preoperative anxiety. The occurrence of preoperative anxiety

Table I Demographic and Clinical Data of the Study Population

\begin{tabular}{|c|c|c|c|c|}
\hline & $\begin{array}{c}\text { ALL } \\
(N=263)\end{array}$ & $\begin{array}{c}\text { POD } \\
(N=73)\end{array}$ & $\begin{array}{l}\text { No POD } \\
(N=190)\end{array}$ & $P$ value \\
\hline Age (years) & $74.2(7.3)$ & $80.9(7.7)$ & $71.7(5.3)$ & $<0.001^{\mathrm{a}}$ \\
\hline Male, sex & $74(28.1 \%)$ & $20(27.4 \%)$ & $54(28.4 \%)$ & $0.869^{b}$ \\
\hline BMI $\left(\mathbf{k g ~ m}^{-2}\right)$ & $24.2(3.9)$ & $23.3(4.0)$ & $24.6(3.8)$ & $0.01 \mathrm{I}^{\mathrm{c}}$ \\
\hline Education level & & & & $0.001^{b}$ \\
\hline Illiteracy & 87 (33.1\%) & $37(50.7 \%)$ & $50(26.3 \%)$ & \\
\hline Primary or junior high graduates & $84(31.9 \%)$ & $20(27.4 \%)$ & $64(33.7 \%)$ & \\
\hline High school and college or higher & $92(35.0 \%)$ & $16(21.9 \%)$ & $76(40.0 \%)$ & \\
\hline History of smoking & 37 (14.1\%) & $10(13.7 \%)$ & $27(\mid 4.2 \%)$ & $0.915^{\mathrm{b}}$ \\
\hline Alcohol use & $42(16.0 \%)$ & $13(17.8 \%)$ & $29(\mid 5.3 \%)$ & $0.614^{b}$ \\
\hline \multicolumn{5}{|l|}{ Comorbidities } \\
\hline Hypertension & $90(34.2 \%)$ & $28(38.4 \%)$ & $62(32.6 \%)$ & $0.38 \mathrm{I}^{\mathrm{b}}$ \\
\hline Diabetes & $42(16.0 \%)$ & $15(20.5 \%)$ & 27 (14.2\%) & $0.209^{b}$ \\
\hline Coronary heart disease & $27(10.3 \%)$ & II (I5.I\%) & $16(8.4 \%)$ & $0.112^{b}$ \\
\hline COPD & $4(1.5 \%)$ & $2(2.7 \%)$ & $2(1.1 \%)$ & $0.66 \mathrm{I}^{\mathrm{b}}$ \\
\hline CKD & $2(0.8 \%)$ & I (I.4\%) & I (0.5\%) & $0.930^{\mathrm{b}}$ \\
\hline DVT & $10(3.8 \%)$ & $4(5.5 \%)$ & $6(3.2 \%)$ & $0.602^{b}$ \\
\hline History of cancer & $4(1.5 \%)$ & I (I.4\%) & $3(1.6 \%)$ & $0.66 \mathrm{I}^{\mathrm{b}}$ \\
\hline $\mathrm{CCl}$ & $3.2(1.0)$ & $3.9(0.9)$ & $3.0(0.9)$ & $<0.001^{\mathrm{a}}$ \\
\hline ASA classification & & & & $<0.001^{\mathrm{b}}$ \\
\hline I & $20(7.6 \%)$ & $0(0 \%)$ & $20(10.5 \%)$ & \\
\hline II & $85(32.3 \%)$ & $5(6.8 \%)$ & $80(42.1 \%)$ & \\
\hline III & $158(60.1 \%)$ & $68(93.2 \%)$ & $90(47.4 \%)$ & \\
\hline VAS pain score (at rest) & $3.0(0.8)$ & $3.0(0.8)$ & $2.9(0.8)$ & $0.373^{\mathrm{a}}$ \\
\hline MMSE & $24(2.3)$ & $22.0(2.2)$ & $24.5(2.0)$ & $<0.001^{\mathrm{a}}$ \\
\hline Preoperative anxiety & $40(15.2 \%)$ & $13(17.8 \%)$ & 27 (I4.2\%) & $0.467^{\mathrm{a}}$ \\
\hline \multicolumn{5}{|l|}{ Laboratory tests } \\
\hline Albumin (g/L) & $39.5(4.4)$ & $37.3(4.7)$ & $40.4(4.0)$ & $<0.001^{\mathrm{a}}$ \\
\hline Serum sodium (mmol/L) & $140.2(3.4)$ & $138.9(4.3)$ & $140.6(2.9)$ & $0.007^{\mathrm{a}}$ \\
\hline Hemoglobin (g/dl) & $12.4(6.4)$ & II.5 (2.3) & $12.7(7.4)$ & $0.007^{\mathrm{a}}$ \\
\hline
\end{tabular}

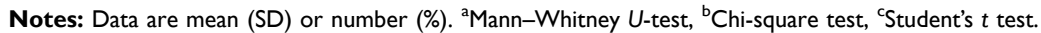

Abbreviations: POD, postoperative delirium; BMI, body mass index; COPD, chronic obstructive pulmonary disease; CKD, chronic kidney disease; DVT, deep vein thrombosis; CCl, Charlson Comorbidity Index; ASA, American Society of Anesthesiologists; VAS, visual analogue scale; MMSE, Mini Mental State Examination. 
Table 2 Surgical Variables and Postoperative Outcomes

\begin{tabular}{|c|c|c|c|c|}
\hline & $\begin{array}{c}\text { ALL } \\
(N=263)\end{array}$ & $\begin{array}{c}\text { POD } \\
(N=73)\end{array}$ & $\begin{array}{l}\text { No POD } \\
(N=\mid 90)\end{array}$ & $P$ value \\
\hline Type of surgery & & & & $0.195^{\mathrm{b}}$ \\
\hline THA & $120(45.6 \%)$ & $38(52.1 \%)$ & $82(43.2 \%)$ & \\
\hline PFNA & $56(21.3 \%)$ & $17(23.3 \%)$ & $39(20.5 \%)$ & \\
\hline TKA & 87 (33.1\%) & $18(24.6 \%)$ & $69(36.3 \%)$ & \\
\hline Duration of anesthesia (min) & $136.6(40.3)$ & $134.3(45.6)$ & I37.5 (38.2) & $0.265^{\mathrm{a}}$ \\
\hline Duration of surgery (min) & $108.2(37.4)$ & $106.6(44.4)$ & $108.9(34.5)$ & $0.237^{\mathrm{a}}$ \\
\hline Blood transfusion & $45(17.1 \%)$ & $18(24.7 \%)$ & $27(\mid 4.2 \%)$ & $0.044^{\mathrm{b}}$ \\
\hline Total fluid administration $(\mathrm{mL})$ & I543 (493) & | 438 (429) & $1583(5 \mid \mathrm{I})$ & $0.039^{\mathrm{a}}$ \\
\hline Nerve block & $15 \mathrm{I}(57.4 \%)$ & 40 (54.8\%) & 111 (58.4\%) & $0.594^{b}$ \\
\hline \multicolumn{5}{|l|}{ Intraoperative medications } \\
\hline Corticosteroid & $59(22.4 \%)$ & $13(17.8 \%)$ & $46(24.2 \%)$ & $0.265^{\mathrm{b}}$ \\
\hline Dexmedetomidine (ug) & $27(18)$ & $28(16)$ & $27(19)$ & $0.796^{\mathrm{a}}$ \\
\hline Benzodiazepines & $219(83.3 \%)$ & 47 (64.4\%) & $172(90.5 \%)$ & $<0.00 \mathrm{I}^{\mathrm{b}}$ \\
\hline PCIA & 209 (79.5\%) & $63(86.3 \%)$ & $146(76.8 \%)$ & $0.265^{\mathrm{b}}$ \\
\hline ICU admission & $53(20.2 \%)$ & $33(45.2 \%)$ & $20(10.5 \%)$ & $<0.00 \mathrm{I}^{\mathrm{b}}$ \\
\hline LOS (days) & $14.2(4.7)$ & $15.5(4.9)$ & I3.7 (4.6) & $0.262^{\mathrm{a}}$ \\
\hline Postoperative LOS (days) & $9.3(4.0)$ & $10.2(4.2)$ & $9.0(3.8)$ & $0.04 I^{\mathrm{a}}$ \\
\hline
\end{tabular}

Notes: Data are mean (SD) or number (\%). ${ }^{a}$ Mann-Whitney U-test, ${ }^{b}$ Chi-square test.

Abbreviations: THA, total hip arthroplasty; PFNA, proximal femoral nail antirotation; TKA, total knee arthroplasty; PCIA, patient-controlled intravenous analgesia; LOS, length of hospital stay.

in THA, PFNA, and TKA was $12.5 \%, 16.1 \%$, and $19.5 \%$, respectively (Figure 2). The number of patients who suffered preoperative anxiety was $13(17.8 \%)$ and $27(14.2 \%)$ in patients with or without POD.

\section{Univariate Analysis}

As is shown in Table 1, patients with POD were older (mean 80.9, $\mathrm{SD}=7.7$ vs mean $71.7, \mathrm{SD}=5.3 ; P<0.001$ ), had lower BMI (mean 23.3, $\mathrm{SD}=4.0$ vs mean 24.6, $\mathrm{SD}=$ 3.8; $P=0.011$ ), $\mathrm{CCI}$ (mean 3.9, $\mathrm{SD}=0.9$ vs mean $3.0, \mathrm{SD}=$ $0.9 ; P<0.001)$, education levels $(P=0.001)$, MMSE (mean $22.0, \mathrm{SD}=2.2$ vs mean $24.5, \mathrm{SD}=2.0 ; P<0.001)$, and higher ASA classification $(P<0.001)$ than patients who did not experience POD. Although some variables were significantly different between patients with and without delirium, preoperative anxiety was not.

In terms of laboratory test results, patients with low level of albumin (mean 37.3, $\mathrm{SD}=4.7$ vs mean $40.4, \mathrm{SD}=$ $4.0 ; P<0.001$ ), serum sodium (mean $138.9, \mathrm{SD}=4.3$ vs mean 140.6, $\mathrm{SD}=2.9 ; P=0.007)$, and hemoglobin concentration (mean 11.5, $\mathrm{SD}=2.3$ vs mean 12.7, $\mathrm{SD}=7.4$;
$P=0.007)$ were more likely to develop delirium. With regard to surgical variables, significant differences were found among the groups in intraoperative blood transfusion $(24.7 \%$ vs $14.2 \% ; P=0.044)$ and total fluid administration (mean 1438, $\mathrm{SD}=429$ vs mean $1583, \mathrm{SD}=511$; $P=0.039)$.

\section{Multivariate Logistic Regression Analysis}

The variables age, BMI, ASA, CCI, education level, MMSE, preoperative anxiety, ICU admission, albumin, serum sodium, hemoglobin, blood transfusion, benzodiazepines, total fluid administration were included in the multivariable logistic model. The forest plot (Figure 3) illustrated that only age $(\mathrm{OR}=1.099,95 \% \mathrm{CI}: 1.013-$ $1.192 ; P=0.023)$, MMSE $(\mathrm{OR}=0.603,95 \% \mathrm{CI}: 0.432-$ $0.842 ; P=0.003)$ and preoperative anxiety $(\mathrm{OR}=3.119$, 95\% CI: $1.144-8.500 ; P=0.026)$ were independently associated with POD. The $\chi^{2}$ value of the Hosmer-Lemeshow test was $10.508(\mathrm{df}=8, P=0.231)$, and the goodness of fit of this model indicated an outstanding fitting. 


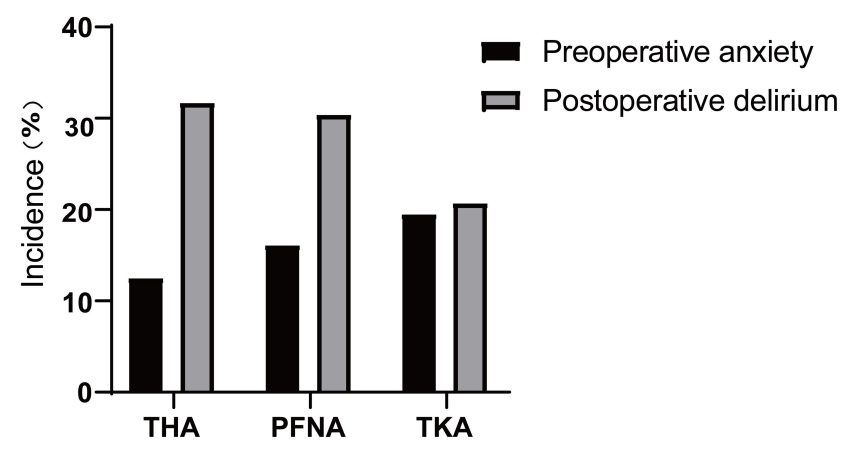

Figure 2 The incidence of preoperative anxiety and postoperative delirium in different types of surgery.

Abbreviations: THA, total hip arthroplasty, PFNA, proximal femoral nail antirotation, TKA, total knee arthroplasty.

\section{Discussion}

We investigated the effects of preoperative anxiety on POD in a cohort of elderly patients undergoing elective orthopedic surgery. In these patients, preoperative anxiety increased the occurrence of POD with an OR 3.119 (1.144-8.500), suggesting that the presence of preoperative anxiety was a significant predictor of POD.
With the rapidly increasing number of older people worldwide, there is a high prevalence of anxiety among older adults, causing detrimental implications from a clinical perspective. Our results show that the total incidence of preoperative anxiety was $15.2 \%$, slightly lower than that of a previous study $(19 \%){ }^{26}$ POD is common, particularly among older people, with a higher incidence in patients scheduled for orthopedic hip or knee surgery. An incidence of POD of $27.8 \%$ was observed. The real frequency may be higher as the presence of delirium was measured only once daily. Due to the fluctuating nature of delirium symptoms, possible cases might have been missed. There is another reason for this-the lower pain score. What is known to us is that uncontrolled pain in elderly patients may induce anxiety and delirium. We were informed of the surgical information the day before surgery, thus the pain was well managed since admission. Additional postoperative pain control was provided for most patients using patient-controlled intravenous analgesia (PCIA).

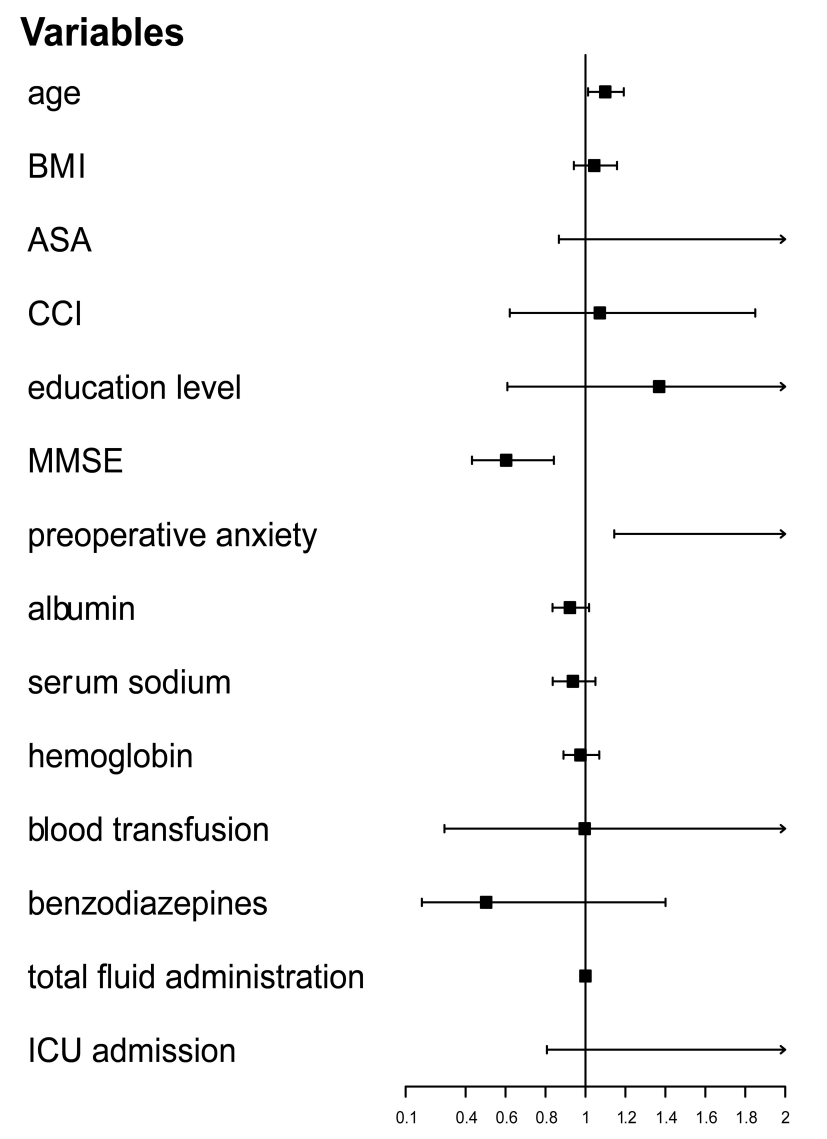

\begin{tabular}{cc} 
OR $(95 \% \mathrm{Cl})$ & P-value \\
$1.099(1.013,1.192)$ & 0.023 \\
$1.044(0.942,1.158)$ & 0.412 \\
$2.597(0.867,7.774)$ & 0.088 \\
$1.072(0.621,1.850)$ & 0.803 \\
$1.369(0.609,3.082)$ & 0.447 \\
$0.603(0.432,0.842)$ & 0.003 \\
$3.119(1.144,8.500)$ & 0.026 \\
$0.922(0.835,1.018)$ & 0.107 \\
$0.937(0.836,1.050)$ & 0.260 \\
\hline $0.975(0.890,1.069)$ & 0.592 \\
\hline $0.996(0.294,3.372)$ & 0.995 \\
\hline $0.503(0.181,1.401)$ & 0.189 \\
\hline $1.000(0.998,1.001)$ & 0.410 \\
\hline $2.112(0.807,5.527)$ & 0.128 \\
\hline & \\
\hline
\end{tabular}

Figure 3 Forest plot of multivariate logistic regression analysis demonstrating factors associated with POD. 
There is a growing body of literature that recognizes the risk factors of POD. Our findings are also consistent with similar research on cancer patients. ${ }^{13}$ However, Van Grootven et al found no relation between preoperative anxiety and POD among 86 elderly hip fracture patients. ${ }^{16}$ Compared to the previous study, the methodological strengths of our study include the prospective design and the relatively large sample size.

Several plausible reasons may explain the close connection between preoperative anxiety and POD. On one hand, in our study, the patients tended to experience acute or chronic pain before admission due to fracture, femoral head necrosis, or osteoarthritis. People with chronic pain are more likely to develop anxiety disorders. ${ }^{27}$ Those suffering from anxiety disorders are associated with decreased quality and quantity of sleep during hospitalization and sleep deprivation has been regarded as a contributing factor to the development of delirium. ${ }^{28}$ On the other hand, anxiety may also be linked to high cortisol levels. Cortisol is known as the stress hormone, mobilizing, and regulating the body's stress response. However, high cortisol levels could be harmful and cause acute cognitive and neuropsychiatric dysfunction. Elevated cortisol levels are correlated with increased risk of POD in patients undergoing CABG surgery ${ }^{29}$ and hip fracture surgery. ${ }^{30}$ Finally, a lot of literature points to a significant correlation between inflammatory cytokine and clinical anxiety. While the pathophysiological mechanisms of delirium remain unclear, inflammatory cytokine has been shown to induce the clinical picture of delirium. ${ }^{31}$

Delirium is a healthcare quality indicator in older adults, and therefore delirium prevention is an essential parameter for patients' safety. ${ }^{32}$ An increasing number of studies investigated the pharmacological and non-pharmacological strategies to prevent delirium. The role of pharmacological strategies, however, remains unclear. ${ }^{10}$ In our study, we collected clinical data regard to medications in operation, including benzodiazepines, dexmedetomidine, and corticosteroid. It is not surprising that no significant difference was found in intraoperative medications between patients with and without POD in the multivariate analysis. In postoperative phase, a recent meta-analysis shows that the use of antipsychotics can successfully reduce the incidence of POD. ${ }^{33}$

Taking into account a large number of trials with a negative conclusion, it seems that a single intervention alone cannot prevent or reduce delirium. There is strong evidence that multi-component delirium prevention interventions are effective in reducing delirium incidence in different hospital settings. ${ }^{10,34}$ Janssen, T. L et al showed that a multimodal prehabilitation program including homebased training exercises, dietary advice and nutritional support, and intravenous iron injections can reduce the occurrence of delirium. ${ }^{35}$ The Hospital Elder Life Program (HELP) provides patients with a screening on admission for six modifiable clinical risk factors (cognitive impairment, sleep deprivation, immobility, vision impairment, hearing impairment, and dehydration). To prevent delirium, a variety of non-pharmacological interventions are implemented for sleep and anxiety. Our findings of the multivariate analysis are in line with the risk factors above. Consequently, the psychological state of patients should also be evaluated preoperatively, which allows us to identify those at high risk. Individualized prevention and treatment interventions need to be performed to minimize the incidence of delirium subsequently.

There were several limitations in this study. First, the results may be imprecise because of the relatively wide confidence intervals around the OR point estimate. Second, we evaluated anxiety on the day before surgery which means it is difficult to judge whether or not they suffered clinical anxiety on admission. It is of clinical significance to recognize anxiety and perform an intervention program to alleviate anxiety as early as possible. Third, only patients undergoing THA, PFNA, and TKA surgery were included and the results cannot be generalized to patients with other surgical types. Fourth, although we adjusted for numerous potential confounders in the analysis, the possibility of unmeasured factors such as depression and postoperative infection must be recognized, which is always a concern in an observational study. Finally, our study did not conduct in-depth analyses for reasons underlying preoperative anxiety. Further studies are necessary for investigating specific reasons to take individualized strategies. Despite these limitations, the study certainly adds to our understanding of the role of anxiety in the development of delirium.

\section{Conclusion}

In conclusion, the current study reveals that preoperative anxiety helps to predict the risk of POD in elderly patients undergoing elective orthopedic surgery. Relieving preoperative anxiety could be a new target for preventive interventions to reduce POD. 


\section{Data Sharing Statement}

The datasets used and analyzed during the current study are available from the corresponding author on reasonable request.

\section{Acknowledgments}

This work was supported by the Department of Anesthesiology, Affiliated Hospital of Xuzhou Medical University.

\section{Disclosure}

The authors report no conflicts of interest in this work.

\section{References}

1. Mu D-L, Zhang D-Z, Wang D-X, et al. Parecoxib supplementation to morphine analgesia decreases incidence of delirium in elderly patients after hip or knee replacement surgery: a randomized controlled trial. Anesth Analg. 2017;124(6):1992-2000. doi:10.1213/ ANE.0000000000002095

2. Bruce AJ, Ritchie CW, Blizard R, Lai R, Raven P. The incidence of delirium associated with orthopedic surgery: a meta-analytic review. Int Psychogeriatr. 2007;19(2):197-214. doi:10.1017/ S104161020600425X

3. Sprung J, Roberts RO, Weingarten TN, et al. Postoperative delirium in elderly patients is associated with subsequent cognitive impairment. $\mathrm{Br}$ J Anaesth. 2017;119(2):316-323. doi:10.1093/bja/aex130

4. Gleason LJ, Schmitt EM, Kosar CM, et al. Effect of delirium and other major complications on outcomes after elective surgery in older adults. JAMA Surg. 2015;150(12):1134-1140. doi:10.1001/ jamasurg.2015.2606

5. Leslie DL, Marcantonio ER, Zhang Y, Leo-Summers L, Inouye SK. One-year health care costs associated with delirium in the elderly population. Arch Intern Med. 2008;168(1):27-32. doi:10.1001/ archinternmed.2007.4

6. Shi Z, Mei X, Li C, et al. Postoperative delirium is associated with long-term decline in activities of daily living. Anesthesiology. 2019;131(3):492-500. doi:10.1097/ALN.0000000000002849

7. Aldecoa C, Bettelli G, Bilotta F, et al. European Society of Anaesthesiology evidence-based and consensus-based guideline on postoperative delirium. Eur J Anaesthesiol. 2017;34(4):192-214.

8. Inouye SK. Delirium in older persons. N Engl J Med. 2006;354 (11):1157-1165. doi:10.1056/NEJMra052321

9. Inouye SK, Westendorp RGJ, Saczynski JS. Delirium in elderly people. Lancet (London, England). 2014;383(9920):911-922. doi:10.1016/S0140-6736(13)60688-1

10. Siddiqi N, Harrison JK, Clegg A, et al. Interventions for preventing delirium in hospitalised non-ICU patients. Cochrane Database Syst Rev. 2016;3:Cd005563. doi:10.1002/14651858.CD005563.pub3

11. Inouye SK, Bogardus ST, Baker DI, Leo-Summers L, Cooney LM. The Hospital Elder Life Program: a model of care to prevent cognitive and functional decline in older hospitalized patients. Hospital Elder Life Program. J Am Geriatr Soc. 2000;48(12):1697-1706.

12. Leung JM, Sands LP, Mullen EA, Wang Y, Vaurio L. Are preoperative depressive symptoms associated with postoperative delirium in geriatric surgical patients? J Gerontol a Biol Sci Med Sci. 2005;60 (12):1563-1568.

13. Wada S, Inoguchi H, Sadahiro R, et al. Preoperative anxiety as a predictor of delirium in cancer patients: a prospective observational cohort study. World J Surg. 2019;43(1):134-142. doi:10.1007/ s00268-018-4761-0
14. Kosar CM, Tabloski PA, Travison TG, et al. Effect of preoperative pain and depressive symptoms on the risk of postoperative delirium: a prospective cohort study. Lancet Psychiatry. 2014;1(6):431-436. doi:10.1016/S2215-0366(14)00006-6

15. Detroyer E, Dobbels F, Verfaillie E, Meyfroidt G, Sergeant P, Milisen K. Is preoperative anxiety and depression associated with onset of delirium after cardiac surgery in older patients? A prospective cohort study. $J$ Am Geriatr Soc. 2008;56 (12):2278-2284. doi:10.1111/j.1532-5415.2008.02013.x

16. Van Grootven B, Detroyer E, Devriendt E, et al. Is preoperative state anxiety a risk factor for postoperative delirium among elderly hip fracture patients? Geriatr Gerontol Int. 2016;16(8):948-955. doi:10.1111/ggi.12581

17. Shin JE, Kyeong S, Lee JS, et al. A personality trait contributes to the occurrence of postoperative delirium: a prospective study. $B M C$ Psychiatry. 2016;16(1):371. doi:10.1186/s12888-016-1079-z

18. Milisen K, Van Grootven B, Hermans W, et al. Is preoperative anxiety associated with postoperative delirium in older persons undergoing cardiac surgery? Secondary data analysis of a randomized controlled trial. BMC Geriatr. 2020;20(1):478. doi:10.1186/s12877-020-01872-6

19. Andreescu C, Lee S. Anxiety disorders in the elderly. Adv Exp Med Biol. 2020;1191:561-576.

20. Ramsay MA. A survey of pre-operative fear. Anaesthesia. 1972;27 (4):396-402. doi:10.1111/j.1365-2044.1972.tb08244.x

21. von Elm E, Altman DG, Egger M, Pocock SJ, Gøtzsche PC, Vandenbroucke JP. The Strengthening the Reporting of Observational Studies in Epidemiology (STROBE) statement: guidelines for reporting observational studies. J Clin Epidemiol. 2008;61 (4):344-349. doi:10.1016/j.jclinepi.2007.11.008

22. Zigmond AS, Snaith RP. The hospital anxiety and depression scale. Acta Psychiatr Scand. 1983;67(6):361-370. doi:10.1111/j.16000447.1983.tb09716.x

23. Zhenxiao S, Huaxue L, Linying J, Tao Z, Luoning Y, Jinyun F. Reliability and validity of hospital anxiety and depression scale. Chin J Clin. 2017;2:11.

24. Inouye SK, van Dyck $\mathrm{CH}$, Alessi CA, Balkin S, Siegal AP, Horwitz RI. Clarifying confusion: the confusion assessment method. A new method for detection of delirium. Ann Intern Med. 1990;113 (12):941-948. doi:10.7326/0003-4819-113-12-941

25. Ely EW, Inouye SK, Bernard GR, et al. Delirium in mechanically ventilated patients: validity and reliability of the confusion assessment method for the intensive care unit (CAM-ICU). JAMA. 2001;286(21):2703-2710. doi:10.1001/jama.286.21.2703

26. Duivenvoorden T, Vissers MM, Verhaar JA, et al. Anxiety and depressive symptoms before and after total hip and knee arthroplasty: a prospective multicentre study. Osteoarthritis Cartilage. 2013;21 (12):1834-1840. doi:10.1016/j.joca.2013.08.022

27. McWilliams LA, Cox BJ, Enns MW. Mood and anxiety disorders associated with chronic pain: an examination in a nationally representative sample. Pain. 2003;106(1-2):127-133. doi:10.1016/S03043959(03)00301-4

28. Watson PL, Ceriana P, Fanfulla F. Delirium: is sleep important? Best Pract Res Clin Anaesthesiol. 2012;26(3):355-366. doi:10.1016/j. bpa.2012.08.005

29. Mu DL, Wang DX, Li LH, et al. High serum cortisol level is associated with increased risk of delirium after coronary artery bypass graft surgery: a prospective cohort study. Crit are (London, England). 2010;14(6):R238. doi:10.1186/cc9393

30. Pearson A, de Vries A, Middleton SD, et al. Cerebrospinal fluid cortisol levels are higher in patients with delirium versus controls. BMC Res Notes. 2010;3:33. doi:10.1186/1756-0500-3-33

31. Cerejeira J, Firmino H, Vaz-Serra A, Mukaetova-Ladinska EB. The neuroinflammatory hypothesis of delirium. Acta Neuropathol. 2010;119(6):737-754. doi:10.1007/s00401-010-0674-1 
32. Sánchez A, Thomas C, Deeken F, et al. Patient safety, cost-effectiveness, and quality of life: reduction of delirium risk and postoperative cognitive dysfunction after elective procedures in older adults-study protocol for a stepped-wedge cluster randomized trial (PAWEL Study). Trials. 2019;20(1):71. doi:10.1186/s13063-0183148-8

33. Janssen TL, Alberts AR, Hooft L, Mattace-Raso F, Mosk CA, van der Laan L. Prevention of postoperative delirium in elderly patients planned for elective surgery: systematic review and meta-analysis. Clin Interv Aging. 2019;14:1095-1117. doi:10.2147/CIA.S201323
34. Martínez F, Donoso AM, Marquez C, Labarca E. Implementing a multicomponent intervention to prevent delirium among critically Ill patients. Crit Care Nurse. 2017;37(6):36-46. doi:10.4037/ $\operatorname{ccn} 2017531$

35. Janssen TL, Steyerberg EW, Langenberg JCM, et al. Multimodal prehabilitation to reduce the incidence of delirium and other adverse events in elderly patients undergoing elective major abdominal surgery: an uncontrolled before-and-after study. PLoS One. 2019;14(6): e0218152. doi:10.1371/journal.pone.0218152

\section{Publish your work in this journal}

Clinical Interventions in Aging is an international, peer-reviewed journal focusing on evidence-based reports on the value or lack thereof of treatments intended to prevent or delay the onset of maladaptive correlates of aging in human beings. This journal is indexed on PubMed Central, MedLine, CAS, Scopus and the Elsevie
Bibliographic databases. The manuscript management system is completely online and includes a very quick and fair peer-review system, which is all easy to use. Visit http://www.dovepress.com/ testimonials.php to read real quotes from published authors. 